\title{
Passive Quasi-free States of the CAR Algebra with Discrete Hamiltonians
}

\author{
By
}

Charles J. K. BATTY*

\begin{abstract}
The spectrally passive, gauge-invariant, quasi-free stases $\omega$ on the $\mathrm{C}^{*}$-algebra of anticommutation relations with respect to a one-parameter quasi-free action $\tau$ are described. If the one-particle Hamiltonian $H$ is discrete, the precise condition on the one-particle density $Q$ of $\omega$ is combinatorial, but if the Connes spectrum of $\tau$ is non-zero, it implies that $Q=\left(I+e^{\beta(H+T)}\right)^{-1}$ for some $\beta \geq 0$ and some operator $T$ of bounded trace norm, apart from some degenerate possibilities. If $H$ has both discrete and continuous parts, these results can be combined with those of de Cannière for the purely continuous case.
\end{abstract}

\section{$\S 1$. Introduction}

The notion of spectral passivity of an invariant state $\omega$ of a $\mathrm{C}^{*}$ algebra $\mathfrak{A}$ with respect to a one-parameter group of automorphisms of $\mathfrak{A}$ was introduced by de Cannière [3] as a part of the KMS condition. Thus $\omega$ is spectrally passive if

$$
\omega\left(x^{*} x\right) \leq \omega\left(x x^{*}\right)
$$

for all $x$ in the spectral subspace $R(-\infty, 0)$ of $\mathfrak{X}$. All KMS states at inverse temperature $\beta$, where $0 \leq \beta \leq \infty$, are spectrally passive. Conversely, the condition

$$
x_{i} \in R\left(-\infty, \lambda_{i}\right), \sum_{i=1}^{n} \lambda_{i} \leq 0 \Rightarrow \prod_{i=1}^{n} \omega\left(x_{i}^{*} x_{i}\right) \leq \prod_{i=1}^{n} \omega\left(x_{i} x_{i}^{*}\right)
$$

implies that $\omega$ is a KMS state at some $0 \leq \beta \leq \infty$ (see [1] for a short proof). The condition (1.1) is closely related to the condition of passivity, as defined by Pusz and Woronowicz [7] based on the

Communicated by H. Araki, October 11, 1985.

* Department of Mathematics, University of Edinburgh, King's Buildings, Edinburgh EH9 3JZ, Scotland, U. K.

Address from 1 October 1985: St. John's College, Oxford OX1 3JP, England. 
Second Law of Thermodynamics. Passive states are spectrally passive [3], and there is no known example of a spectrally passive state which is not passive (see [1] for some partial results).

Now de Cannière [4] has investigated the condition (1.1) in the specific context of a (gauge-invariant) quasi-free state and quasifree automorphisms on the CAR algebra $\mathscr{U}(\mathscr{H})$ associated with a one-particle Hilbert space $\mathscr{H}$ (or the gauge-invariant part $\mathfrak{U}(\mathscr{H})^{(0)}$ ). Thus $\mathfrak{A}(\mathscr{H})$ is generated by annihilation and creation operators $a(f)$, $a^{*}(g)(f, g \in \mathscr{H})$ satisfying the canonical anticommutation relations

$$
\begin{aligned}
& a(f) a(g)+a(g) a(f)=0 \\
& a(f) a^{*}(g)+a^{*}(g) a(f)=(f, g) I .
\end{aligned}
$$

These conditions, together with linearity of $a^{*}$ and the relation $a^{*}(f)$ $=(a(f))^{*}$, determine $\mathfrak{X}(\mathscr{H})$ uniquely up to isomorphism. The quasifree one-parameter group of automorphisms is given by

$$
\tau_{t}(a(f))=a\left(e^{i t H} f\right)
$$

where $e^{i t H}$ is a unitary group on $\mathscr{H}$ with one-particle Hamiltonian $H$. The gauge action is the quasi-free one-parameter action with $H=I$. The gauge-invariant quasi-free states $\omega$ are in one-to-one correspondence with the positive contractions $Q$ on $\mathscr{H}$, the correspondence being given by

$$
\omega\left(a^{*}\left(g_{1}\right) \ldots \ldots a^{*}\left(g_{n}\right) a\left(f_{n}\right) \ldots \ldots a\left(f_{1}\right)\right)=\operatorname{det}\left[\left(f_{i}, Q g_{j}\right)\right] .
$$

The operator $Q$ is known as the one-particle density of $\omega$. Invariance of $\omega$ under $\tau$ corresponds to $Q$ commuting with $H$. All these, and other, properties of $\mathfrak{U}(\mathscr{H})$ may be found in [2,5.2].

It was shown in [4] that if $H$ has no eigenvectors then $\omega$ is spectrally passive on $\mathfrak{U}(\mathscr{H})^{(0)}$ if and only if either $Q=P(-\infty, \mu)$ for some $-\infty \leq \mu \leq \infty$, where $P$ is the spectral measure of $H$, or $Q=(I$ $\left.+e^{\beta H-\mu I}\right)^{-1}$ for some $\beta \geq 0$ and some $-\infty<\mu<\infty$. Furthermore, if $\omega$ is spectrally passive on $\mathscr{U}(\mathscr{H})$, then $Q=P(-\infty, 0)$ or $Q=\left(I+e^{\beta H}\right)^{-1}$. Some partial results were given in [4] if $H$ has some eigenvectors.

This paper continues this programme by considering all possible combinations of eigenvalues of $H$. Thus a complete description is given in Theorem 4.3 (see Corollary 5.2 for the converse result) of all the one-particle densities $Q$ whose associated states are spectrally passive on $\mathfrak{U}(\mathscr{H})$. If $H$ is diagonalisable (so that $H$ has a complete orthonormal set of eigenvectors), then $Q$ is also diagonal- 
isable, and the eigenvalues of $H, Q$ are paired in a certain combinatorial way (the "passive" pairs of $\S 3$ ). If the eigenvalues of $H$ are evenly spaced or of large multiplicity, this requires that $Q=(I$ $\left.+e^{\beta(H+T)}\right)^{-1}$ for some $\beta \geq 0$ and some operator $T$ of trace class, apart from some degenerate possibilities. The assumptions on the eigenvalues are essentially that the Connes spectrum $\Gamma(\tau)$ of $\tau$ should not be (0), and the conclusion is that $T=0$ if $\Gamma(\tau)=\mathbb{R}$ and $\operatorname{tr}|T|$ $\leq \gamma$ if $\Gamma(\tau)=\gamma \mathbb{Z}$ for some $\gamma>0$. If $H$ has some eigenvectors but is not diagonalisable, then either $Q=\left(I+e^{\beta H}\right)^{-1}$ or $Q(I-P(0))=P(-\infty$, 0 ) or $H$ has a direct sum decomposition $H=H_{1} \oplus H_{2}$ where $H_{1}$ is of trace class and $\left(-\operatorname{tr}\left|H_{1}\right|, \operatorname{tr}\left|H_{1}\right|\right)$ is contained in the resolvent set of $H_{2}$, and $Q=Q_{1} \oplus P_{2}(-\infty, 0)$, where $\left(H_{1}, Q_{1}\right)$ is a passive pair and $P_{2}$ is the spectral measure of $H_{2}$. In the final section, corresponding results for $\mathscr{A}(\mathscr{H})^{(0)}$ are stated.

The techniques of proof in this paper are mostly derived from [4], with certain extra combinatorial complications.

\section{§. Exponentials of Trace Class Operators}

In the sequel, it will be seen that spectral passivity of $\omega$ implies inequalities of the form

$$
\prod_{i=1}^{m}\left(f_{i}, Q f_{i}\right) \prod_{j=m+1}^{n}\left(1-\left(f_{j}, Q f_{j}\right)\right) \leq c \prod_{i=1}^{m}\left(1-\left(f_{i}, Q f_{i}\right)\right) \prod_{j=m+1}^{n}\left(f_{j}, Q f_{j}\right)
$$

for certain constants $c$, integers $0 \leq m \leq n$ and orthonormal sets $\left\{f_{i}\right.$ : $1 \leq i \leq n\}$ with $\left(f_{i}, Q f_{j}\right)=0$ for $1 \leq i<j \leq n$. The results in this section describe some of the consequences of (2.1). For an operator $T$ on $\mathscr{H}$, let $\|T\|_{1}=\operatorname{tr}|T|$ if $T$ is of trace class, $\|T\|_{1}=\infty$ otherwise.

Lemma 2.1. Let $Q$ be a positive contraction on $\mathscr{H}$ such that $Q$ and $I-Q$ are invertible, and let $b$ be a positive real number. The following are equivalent :

(i) $\log \left(b\left(Q^{-1}-I\right)\right)$ is of trace class,

(ii) $Q-b(b+1)^{-1} I$ is of trace class,

(iii) $Q$ has a complete orthonormal set of eigenvectors $\left\{f_{\alpha}: \alpha \in A\right\}$ with eigenvalues $\rho_{\alpha}$, and there are constants $0<c_{1} \leq 1 \leq c_{2}<\infty$ such that

$$
c_{1} \leq \prod_{\alpha \in B} b\left(\rho_{\alpha}^{-1}-1\right) \leq c_{2}
$$


In this case,

for all finite subsets $B$ of $A$.

$$
\begin{array}{r}
\exp \left(\left\|\log \left(b\left(Q^{-1}-I\right)\right)\right\|_{1}\right)=\sup \left\{\prod_{\alpha \in A_{1}} b\left(\rho_{\alpha}^{-1}-1\right) / \prod_{\alpha \in A_{2}} b\left(\rho_{\alpha}^{-1}-1\right)\right\} \\
=\prod_{\rho_{\alpha}<b(b+1)^{-1}} b\left(\rho_{\alpha}^{-1}-1\right) / \prod_{\rho_{\alpha}>b(b+1)^{-1}} b\left(\rho_{\alpha}^{-1}-1\right)
\end{array}
$$

where the supremum in (2.3) is taken over all finite (disjoint) subsets $A_{1}, A_{2}$ of $A$.

Proof. The equivalence of (i) and (ii) follows from the inequality

$$
4\left|t-b(b+1)^{-1}\right| \leq\left|\log \left(b\left(t^{-1}-1\right)\right)\right| \leq \varepsilon^{-1}(1-\varepsilon)^{-1}\left|t-b(b+1)^{-1}\right|
$$

which is valid for $\varepsilon \leq t \leq 1-\varepsilon, 0<\varepsilon \leq \min (1, b) /(b+1)$.

The equivalence of (i) and (iii) follows from the fact that (2.2) is equivalent to the condition

$$
\sum_{\alpha \in A} \mid \log \left(b\left(\rho_{\alpha}^{-1}-1\right) \mid \leq \log \left(c_{2} / c_{1}\right) .\right.
$$

This also establishes (2.3) and (2.4).

Lemma 2. 2. Let $Q$ be a positive contraction on $\mathscr{H}, Q \neq 0, I$.

1. Suppose that there is a constant $c \geq 1$ such that (2.1) is valid for all integers $0 \leq m \leq n$ and orthonormal sets $\left\{f_{i}: 1 \leq i \leq n\right\}$ with $\left(f_{i}, Q f_{j}\right)=0$ for $1 \leq i<j \leq n$. Then $Q$ and $I-Q$ are invertible, and $\left\|\log \left(Q^{-1}-I\right)\right\|_{1} \leq$ $\log c$.

2. Suppose that there is a constant $c \geq 1$ such that (2.1) is valid for $n=2 m \geq 0$, and for all orthonormal sets $\left\{f_{i}: 1 \leq i \leq 2 m\right\}$ with $\left(f_{i}, Q f_{j}\right)$ $=0$ for $1 \leq i<j \leq 2 m$. Then $Q$ and $I-Q$ are invertible, and there exists $b$ $>0$ such that $\left\|\log \left(b\left(Q^{-1}-I\right)\right)\right\|_{1} \leq \log c$.

The conclusions of (1) and (2) remain valid if $\mathscr{H}$ has a complete orthonormal set of eigenvectors for $Q$ and (2.1) is assumed only to hold when each $f_{i}$ belongs to this set.

Proof. 1. Taking $m=0, n=1$ in (2.1) shows that $Q$ is invertible (and $\left.Q \geq(c+1)^{-1} I\right)$. Taking $m=1, n=1$ shows that $I-Q$ is invertible (and $\left.Q \leq c(c+1)^{-1} I\right)$.

Suppose that $Q-\frac{1}{2} I$ is not compact. By spectral theory, there exist $\varepsilon>0$ and an infinite orthonormal sequence $\left\{f_{i}\right\}$ such that $\left(f_{i}, Q f_{j}\right)=$ 
0 if $\mathrm{i} \neq j$, and either $\left(f_{i}, Q f_{i}\right) \leq \frac{1}{2}-\varepsilon$ for all $i$, or $\left(f_{i}, Q f_{i}\right) \geq \frac{1}{2}+\varepsilon$ for all i. Putting $m=0$ or $m=n$ in (2.1), it follows that

$$
\left(\frac{1}{2}+\varepsilon\right)^{n} \leq c\left(\frac{1}{2}-\varepsilon\right)^{n}
$$

for all n. This contradiction shows that $Q-\frac{1}{2} I$ is compact, and in particular $Q$ has a complete set of eigenvectors $\left\{\mathrm{g}_{\alpha}: \alpha \in A\right\}$ with corresponding eigenvalues $\rho_{\alpha}$. If $A_{1}$ and $A_{2}$ are finite disjoint subsets of $A$, then (2.1) gives

$$
\prod_{\alpha \in A_{1}}\left(\rho_{\alpha}^{-1}-1\right) / \prod_{\alpha \in A_{2}}\left(\rho_{\alpha}^{-1}-1\right) \leq c,
$$

so $\left\|\log \left(Q^{-1}-I\right)\right\|_{1} \leq \log c$, by Lemma 2.1.

2. If $Q$ were not injective, there would be orthonormal vectors $f_{1}$, $f_{2}$ with $Q f_{1} \neq 0, Q f_{2}=0$. Applying (2.1) with $m=1, n=2$ would lead to a contradiction. Similarly, $I-Q$ is injective.

Suppose that $Q-b^{\prime} I$ is not compact for any $b^{\prime}$. By spectral theory, there exist $0<b_{1}<b_{2}<1$ and infinite orthonormal sequences $\left\{f_{i}\right\}$ and $\left\{g_{j}\right\}$ such that

$$
\begin{aligned}
& \left(f_{i}, Q f_{j}\right)=\left(g_{i}, Q g_{j}\right)=0 \quad \text { if } i \neq j \\
& \left(f_{i}, g_{j}\right)=\left(f_{i}, Q g_{j}\right)=0 \quad \text { for all } i, j \\
& \left(f_{i}, Q f_{i}\right)>b_{2},\left(g_{j}, Q g_{j}\right)<b_{1} \quad \text { for all } i, j_{0}
\end{aligned}
$$

Applying (2.1) to $\left\{f_{1}, \ldots f_{m}, g_{1}, \ldots g_{m}\right\}$,

$$
b_{2}^{m}\left(1-b_{1}\right)^{m} \leq c\left(1-b_{2}\right)^{m} b_{1}^{m} \text { 。 }
$$

Since $b_{2}\left(1-b_{1}\right)>\left(1-b_{2}\right) b_{1}$, this is a contradiction, showing that $Q-b^{\prime} I$ is compact for some $b^{\prime}$, and $Q$ has a complete orthonormal set of eigenvectors $\left\{h_{\alpha}: \alpha \in A\right\}$ with corresponding eigenvalues $\rho_{\alpha^{\circ}}$.

If $\mathscr{H}$ is infinite-dimensional, $b^{\prime}$ is unique, and $0<b^{\prime}<1$. Let $b=$ $b^{\prime}\left(1-b^{\prime}\right)^{-1}$. Let $A_{1}, A_{2}$ be finite disjoint subsets of $A$ with cardinalities $m, n$, respectively, and $A_{3}$ be a subset of $A \backslash\left(A_{1} \cup A_{2}\right)$ of cardinality $|m-n|$ such that $\rho_{\alpha}$ is arbitrarily close to $b^{\prime}$ for all $\alpha$ in $A_{3}$. Applying (2.1) to $\left\{h_{\alpha}: \alpha \in A_{2} \cup A_{3} \cup A_{1}\right\}$ and taking a limit,

$$
\left(b^{\prime}\right)^{m-n} \prod_{\alpha \in A_{2}} \rho_{\alpha} \prod_{\alpha \in A_{1}}\left(1-\rho_{\alpha}\right) \leq c\left(1-b^{\prime}\right)^{m-n} \prod_{\alpha \in A_{2}}\left(1-\rho_{\alpha}\right) \underset{\alpha \in A_{1}}{\rho_{\alpha}}
$$

or

$$
\prod_{\alpha \in A_{1}} b\left(\rho_{\alpha}^{-1}-1\right) / \prod_{\alpha \in A_{2}} b\left(\rho_{\alpha}^{-1}-1\right) \leq c
$$


so $\left\|\log \left(b\left(Q^{-1}-1\right)\right)\right\|_{1} \leq \log c$, by Lemma 2.1 .

If $\mathscr{H}$ is finite-dimensional, choose $b^{\prime}$ so that $Q$ has equal numbers of eigenvalues greater than (respectively, less than) $b^{\prime}$, and let $b=$ $b^{\prime}\left(1-b^{\prime}\right)^{-1}$. In the argument above, consider all the eigenvectors (except possibly one) simultaneously, and deduce that $\| \log \left(b\left(Q^{-1}-\right.\right.$ I)) $\|_{1} \leq \log c$.

The proof of the final statement is included in the proof above.

Remark. There are converse results to Lemma 2.2, obtained by applying the theory of trace class operators to $\log \left(b\left(Q^{-1}-I\right)\right)$. Thus (2.1) holds with $c=\exp \left\|\log \left(b\left(Q^{-1}-I\right)\right)\right\|_{1}$ for any orthonormal set $\left\{f_{i}\right\}$, provided that $m=n$ if $b \neq 1$.

\section{§3. Passive Pairs of Operators}

Let $H$ be a (possibly unbounded) self-adjoint operator on $\mathscr{H}$ with spectral measure $P$, and $Q$ be a positive contraction on $\mathscr{H}$. The pair $(H, Q)$ will be said to be passive if $\mathscr{H}$ has a complete orthonormal set $\left\{f_{\alpha}: \alpha \in A\right\}$ of common eigenvectors for $H, Q$ with eigenvalues $\lambda_{\alpha}, \rho_{\alpha}$ respectively, and

$$
\prod_{\alpha \in A_{1}} \rho_{\alpha} \prod_{\alpha \in A_{2}}\left(1-\rho_{\alpha}\right) \leq \prod_{\alpha \in A_{1}}\left(1-\rho_{\alpha}\right) \prod_{\alpha \in A_{2}} \rho_{\alpha}
$$

for any finite (disjoint) subsets $A_{1}, A_{2}$ of $A$ such that $\lambda\left(A_{1}\right)>\lambda\left(A_{2}\right)$, where $\lambda(B)=\sum_{\alpha \in B} \lambda_{\alpha}$ for a finite subset $B$ of $A$.

If $\rho_{\alpha}=0$ whenever $\lambda_{\alpha}>0$ and $\rho_{\alpha}=1$ whenever $\lambda_{\alpha}<0$, then $(H, Q)$ is a passive pair. Such a passive pair will be said to be trivial.

If $H$ has a complete orthonormal set of eigenvectors, then $(H$, $\left.\left(I+e^{\beta H}\right)^{-1}\right)$ is a passive pair for any $\beta \geq 0$.

If $(H, Q)$ is a passive pair, then (3.1) gives

$$
\begin{aligned}
& \lambda_{\alpha}>0 \Rightarrow 0 \leq \rho_{\alpha} \leq \frac{1}{2} \\
& \lambda_{\alpha}<0 \Rightarrow \frac{1}{2} \leq \rho_{\alpha} \leq 1 \\
& \lambda_{\alpha_{1}}>\lambda_{\alpha_{2}} \Rightarrow \rho_{\alpha_{1}} \leq \rho_{\alpha_{2}} \\
& \lambda_{\alpha_{1}}+\lambda_{\alpha_{2}}>0 \Rightarrow \rho_{\alpha_{1}}+\rho_{\alpha_{2}} \leq 1 \\
& \lambda_{\alpha_{1}}+\lambda_{\alpha_{2}}<0 \Rightarrow \rho_{\alpha_{1}}+\rho_{\alpha_{2}} \geq 1 .
\end{aligned}
$$


Lemma 3.1. Suppose that $(H, Q)$ is a non-trivial passive pair, and let $\mathscr{H}_{0}=P(0) \mathscr{H}, Q_{0}=Q \mid \mathscr{H}_{0}$. Then $Q_{0}$ and $I-Q_{0}$ are invertible and $\log \left(Q_{0}^{-1}-I_{\mathscr{H}_{0}}\right)$ is of trace class on $\mathscr{H}_{0}$. Furthermore

$$
\prod_{\alpha \in A_{1}} \rho_{\alpha} \prod_{\alpha \in A_{2}}\left(1-\rho_{\alpha}\right) \leq \exp \left(-\left\|\log \left(Q_{0}^{-1}-I_{\mathscr{H}_{0}}\right)\right\|_{1}\right) \underset{\alpha \in A_{1}}{\Pi}\left(1-\rho_{\alpha}\right) \prod_{\alpha \in A_{2}} \rho_{\alpha}
$$

for any finite subsets $A_{1}, A_{2}$ of $\left\{\alpha \in A: \lambda_{\alpha} \neq 0\right\}$ such that $\lambda\left(A_{1}\right)>\lambda\left(A_{2}\right)$.

Proof. Since $(H, Q)$ is non-trivial, there is some $\kappa$ in $A$ such that either $\lambda_{\kappa}>0$ and $\rho_{\kappa}>0$ or $\lambda_{\kappa}<0$ and $\rho_{\kappa}<1$. Let $c=\rho_{\kappa}^{-1}-1$ or $c=$ $\left(1-\rho_{k}\right)^{-1}-1$, respectively.

Let $B_{1}$ and $B_{2}$ be finite subsets of $\left\{\alpha \in A: \lambda_{\alpha}=0\right\}$. Applying (3.1) to $A_{1} \cup B_{1}, A_{2} \cup B_{2}$,

$$
\prod_{A_{1}} \rho_{\alpha} \prod_{A_{2}}\left(1-\rho_{\alpha}\right) \prod_{B_{1}} \rho_{\alpha} \underset{B_{2}}{\Pi}\left(1-\rho_{\alpha}\right) \leq \prod\left(1-\rho_{\alpha}\right) \prod_{A_{2}} \rho_{\alpha} \prod_{B_{1}}\left(1-\rho_{\alpha}\right) \prod_{B_{2}} \rho_{\alpha} \text {. }
$$

The special case when $A_{1}=\{\kappa\}, A_{2}=\phi$ or $A_{1}=\phi, A_{2}=\{\kappa\}$ shows that

$$
\prod_{B_{1}} \rho_{\alpha} \prod_{B_{2}}\left(1-\rho_{\alpha}\right) \leq c \prod_{B_{1}}\left(1-\rho_{\alpha}\right) \prod_{B_{2}} \rho_{\alpha} \text { 。 }
$$

By Lemma 2.2(1) applied to $\mathscr{H}_{0}$ and $Q_{0}, Q_{0}$ and $I_{\mathscr{H}_{0}}-Q_{0}$ are invertible, and $\log \left(Q_{0}^{-1}-I_{\mathscr{F}_{0}}\right)$ is of trace class. On rearranging (3.8) and taking an infimum over all choices of $B_{1}, B_{2},(3.7)$ follows.

The inequalities $(3.2),(3.3)$ may now be strengthened to give

$$
\begin{aligned}
& \lambda_{\alpha}>0 \Rightarrow 0 \leq \rho_{\alpha} \leq(1+\delta)^{-1} \\
& \lambda_{\alpha}<0 \Rightarrow \delta(1+\delta)^{-1} \leq \rho_{\alpha} \leq 1
\end{aligned}
$$

where $\delta=\exp \left(\left\|\log \left(Q_{0}^{-1}-1_{\mathscr{C}_{0}}\right)\right\|_{1}\right)$.

Proposition 3.2. Let $(H, Q)$ be a non-trivial passive pair, and suppose that either $Q$ or $I-Q$ is not injective. There is a decomposition $\mathscr{H}=\mathscr{H}_{1} \oplus \mathscr{H}_{2}, H=H_{1} \oplus H_{2}, Q=Q_{1} \oplus P_{2}(-\infty, 0)$, where $P_{2}$ is the spectral measure of $H_{2}, H_{1}$ is of trace class, $\left(-\left\|H_{1}\right\|_{1},\left\|H_{1}\right\|_{1}\right)$ is contained in the resolvent set of $H_{2}, Q_{1}$ and $I_{\mathscr{H}_{1}}-Q_{1}$ are invertible and $\left(H_{1}, Q_{1}\right)$ is a passive pair.

Proof。 Let $A_{1}=\left\{\alpha \in A: 0<\rho_{\alpha}<1\right\}, A_{2}=A \backslash A_{1}, \gamma=\inf \left\{\left|\lambda_{\alpha}\right|: \alpha \in A_{2}\right\}$. 
By assumption, $A_{2}$ is non-empty, so $\gamma$ is finite. It follows from (3.2) $-(3.6)$ that

$$
\left|\lambda_{\alpha}\right|>\gamma \Rightarrow \alpha \in A_{2}
$$

Since $(H, Q)$ is non-trivial, it follows that $\gamma>0$. If $\mathscr{H}_{i}$ is the closed linear span of $\left\{f_{\alpha}: \alpha \in A_{i}\right\}$ then $H=H_{1} \oplus H_{2}, Q=Q_{1} \oplus P_{2}(-\infty, 0)$ (using (3.2), (3.3)), $\left\|H_{1}\right\| \leq \gamma,(-\gamma, \gamma)$ is contained in the resolvent set of $H_{2}, Q_{1}$ and $I_{\mathscr{H}_{1}}-Q_{1}$ are injective, and $\left(H_{1}, Q_{1}\right)$ is a passive pair.

Let $B_{1}, B_{2}$ be finite subsets of $A_{1}$, and suppose that $\lambda\left(B_{1}\right)-\lambda\left(B_{2}\right)$ $>\gamma$. By definition of $\gamma$, there exists an index $\kappa$ in $A_{2}$ such that $\left|\lambda_{\kappa}\right|$ $<\lambda\left(B_{1}\right)-\lambda\left(B_{2}\right)$. Applying (3.1) to $B_{1}, B_{2} \cup\{\kappa\}$ or to $B_{1} \cup\{\kappa\}, B_{2}$ (depending on whether $\lambda_{\kappa}>0$ or $\lambda_{\kappa}<0$,

$$
\prod_{B_{1}} \rho_{\alpha} \prod_{B_{2}}\left(1-\rho_{\alpha}\right) \leq 0
$$

since $\rho_{\kappa}=0$ or $\rho_{\kappa}=1$. This contradicts the definition of $A_{1}$. Hence $\left\|H_{1}\right\|_{1} \leq \gamma$.

Let $\gamma^{\prime}=\max \left\{\left|\lambda_{\alpha}\right|: \alpha \in A_{1}\right\}$. It follows from (3.4)-(3.6) that

$$
\left\|Q_{1}^{-1}\right\|,\left\|\left(I_{\mathscr{H}_{1}}-Q_{1}\right)^{-1}\right\| \leq \max \left\{\rho_{\alpha}^{-1} \bigvee\left(1-\rho_{\alpha}\right)^{-1}: \alpha \in A_{1},\left|\lambda_{\alpha}\right|=\gamma^{\prime}\right\},
$$

the right-hand side being finite since there are only finitely many indices $\alpha$ in $A_{1}$ with $\left|\lambda_{\alpha}\right|=\gamma^{\prime}$.

Proposition 3.2 reduces the problem of describing passive pairs to the case when $Q$ and $I-Q$ are injective. The aim is to show then that

$$
Q=\left(I+e^{\beta(H+T)}\right)^{-1}
$$

for some $\beta \geq 0$ and some trace class operator $T$ with $\|T\|_{1}$ bounded. Thus the problem is to show that

$$
T_{\beta}:=\log \left(Q^{-1}-I\right)-\beta H
$$

is trace class (and to obtain a bound on its trace norm). Writing

$$
\begin{aligned}
\sigma_{\alpha} & =\log \left(\rho_{\alpha}^{-1}-1\right) \\
\sigma(B) & =\sum_{B} \sigma_{\alpha}
\end{aligned}
$$

for a finite subset $B$ of $A,(3.1)$ becomes

$$
\lambda\left(A_{1}\right)>\lambda\left(A_{2}\right) \Rightarrow \sigma\left(A_{1}\right) \geq \sigma\left(A_{2}\right),
$$

and the objective is to show that 


$$
\sup _{A_{1}, A_{2}}\left\{\sigma\left(A_{1}\right)-\sigma\left(A_{2}\right)-\beta\left(\lambda\left(A_{1}\right)-\lambda\left(A_{2}\right)\right)\right\}<\infty .
$$

The obstruction to $(3.13)$ relates to the possibility of the quasifree action $\tau$ on $\mathfrak{A}(\mathscr{H})$ associated with $H$ having zero Connes spectrum. Let $\Gamma(H)$ be the set of all real numbers $\gamma$ such that there are sequences of mutually disjoint finite subsets $A_{n}, B_{n}$ of $A$ such that $\lambda\left(A_{n}\right)-\lambda\left(B_{n}\right) \rightarrow \gamma$. Elementary combinatorial considerations show that $\Gamma(H)$ is a closed subgroup of $\mathbb{R}$. In this case where $H$ is diagonalisable, $\mathfrak{A}(\mathscr{H})$ may be identified with $\bigotimes_{\alpha \in A} M_{2}$ and $\tau$ with

$$
\otimes A d\left[\begin{array}{cc}
1 & 0 \\
0 & e^{i t \lambda} \alpha_{\alpha}
\end{array}\right]
$$

(see $[2,5.2 .5]$ and Section 5). It was observed in [6] that $\Gamma(H)$ is the Connes spectrum of this action.

If $H$ is of trace class, then $\Gamma(H)=(0)$; if $H$ is compact, but not of trace class, then $\Gamma(H)=\mathbb{R}$; if $H$ is bounded but not of trace class, then $\Gamma(H) \neq(0)$; in general, $\Gamma(H)$ contains all eigenvalues of infinite multiplicity.

The first case when it is possible to establish $(3.11)$ is when $H$ itself is of trace class. This result may be applied to the pair $\left(H_{1}\right.$, $Q_{1}$ ) of Proposition 3.2.

Proposition 3. 3. Let $(H, Q)$ be a non-trivial passive pair, where $H$ is of trace class, and $Q$ and $I-Q$ are injective. Then $\log \left(Q^{-1}-I\right)$ is of trace class, and, for any $\beta \geq 0, Q=\left(I+e^{\beta(H+T)}\right)^{-1}$ for some operator $T$ of trace class.

Proof. It is possible to consider separately the restrictions to $\mathrm{P}(0) \mathscr{H}, \mathrm{P}(-\infty, 0) \mathscr{H}$ and $\mathrm{P}(0, \infty) \mathscr{H}$. The first of these is covered by Lemma 3.1, and the other two are similar to each other, so we consider only the latter. Assume therefore that $\lambda_{\alpha}>0$ for all $\alpha$, so that $\sigma_{\alpha} \geq 0$ by (3.2). Let $A_{1}$ be a finite subset of $A$ such that $\lambda\left(A_{1}\right)>$ $\frac{1}{2}\|H\|_{1}$, and $A_{2}$ be any finite subset of $A \backslash A_{1}$. Then $\lambda\left(A_{1}\right)>\lambda\left(A_{2}\right)$, so by (3.12) $\sigma\left(A_{1}\right) \geq \sigma\left(A_{2}\right)$. Thus $\sum_{A} \sigma_{\alpha} \leq 2 \sigma\left(A_{1}\right)$, as required.

The next case is when $\Gamma(H) \neq(0)$.

Proposition 3.4. Let $(H, Q)$ be a passive pair, and suppose that $Q$ 
and $I-Q$ are injective.

1. If $\Gamma(H)=\gamma \mathbb{Z}$ where $\gamma>0$, then $Q=\left(I+e^{\beta(H+T)}\right)^{-1}$ for some $\beta \geq 0$ and some operator $T$ with $\|T\|_{1} \leq \gamma$.

2. If $\Gamma(H)=\mathbb{R}$, then $Q=\left(I+e^{\beta H}\right)^{-1}$ for some $\beta \geq 0$.

Proof. Let $\gamma>0$ be any member of $\Gamma(H)$, and $A_{n}, B_{n}(n \geq 1)$ be disjoint finite subsets of $A$ such that $\lambda\left(A_{n}\right)-\lambda\left(B_{n}\right) \rightarrow \gamma$. By (3.12), $\sigma\left(A_{n}\right)-\sigma\left(B_{n}\right) \geq 0$ for large $n$.

Let $\alpha$ be any index with $\lambda_{\alpha}>0$. Choose $m$ so that $\alpha \notin A_{m} \cup B_{m}$, $\lambda\left(A_{m}\right)-\lambda\left(B_{m}\right)>\gamma-\lambda_{\alpha}$. If $\alpha \notin A_{n} \cup B_{n}, n \neq m$, then

$$
\begin{aligned}
\lambda\left(A_{m} \cup B_{n} \cup\{\alpha\}\right) & =\lambda\left(A_{m}\right)+\lambda\left(B_{n}\right)+\lambda_{\alpha} \\
& >\lambda\left(B_{m}\right)+\lambda\left(A_{n}\right) \\
& =\lambda\left(B_{m} \cup A_{n}\right)
\end{aligned}
$$

if $n$ is large. By (3. 12)

$$
\sigma\left(A_{m}\right)+\sigma\left(B_{n}\right)+\sigma_{\alpha} \geq \sigma\left(B_{m}\right)+\sigma\left(A_{n}\right) .
$$

Thus

$$
0 \leq \sigma\left(A_{n}\right)-\sigma\left(B_{n}\right) \leq \sigma\left(A_{m}\right)-\sigma\left(B_{m}\right)+\sigma_{\alpha} .
$$

If $\lambda_{\alpha}<0$, a similar argument shows that

$$
0 \leq \sigma\left(A_{n}\right)-\sigma\left(B_{n}\right) \leq \sigma\left(A_{m}\right)-\sigma\left(B_{m}\right)-\sigma_{\alpha} \text { 。 }
$$

Since $H \neq 0$, choosing $\alpha$ so that $\lambda_{\alpha} \neq 0$, it follows in either case that $\sigma\left(A_{n}\right)-\sigma\left(B_{n}\right)$ is bounded. Passing to a subsequence, assume that $\sigma\left(A_{n}\right)-\sigma\left(B_{n}\right)$ converges to a limit $\beta \gamma$, where $\beta \geq 0$.

Now let $A_{0}, B_{0}$ be disjoint finite subsets of $A$, and put $\lambda\left(A_{0}\right)-$ $\lambda\left(B_{0}\right)=\gamma_{0}$. If $\gamma_{0}<0$, let $k \geq 0$ be the largest integer such that $k \gamma+\gamma_{0}<0$. For large $n$, the sets $A_{0}, B_{0}, A_{n}, B_{n}, A_{n+1}, B_{n+1}, \ldots$ are disjoint. Let $C_{n}=A_{0} \cup A_{n} \cup \ldots \cup A_{n+k-1}, D_{n}=B_{0} \cup B_{n} \cup \ldots \cup B_{n+k-1}$. Then

$$
\lambda\left(C_{n}\right)-\lambda\left(D_{n}\right)=\gamma_{0}+\sum_{i=n}^{n+k-1}\left(\lambda\left(A_{i}\right)-\lambda\left(B_{i}\right)\right)<0
$$

for large $n$. By $(3.12)$

$$
\begin{aligned}
0 \geq \sigma\left(C_{n}\right)-\sigma\left(D_{n}\right) & =\sigma\left(A_{0}\right)-\sigma\left(B_{0}\right)+\sum_{i=n}^{n+k-1}\left(\sigma\left(A_{i}\right)-\sigma\left(B_{i}\right)\right) \\
& \rightarrow \sigma\left(A_{0}\right)-\sigma\left(B_{0}\right)+k \beta \gamma
\end{aligned}
$$

as $n \rightarrow \infty$. Hence

$$
\sigma\left(A_{0}\right)-\sigma\left(B_{0}\right)-\beta\left(\lambda\left(A_{0}\right)-\lambda\left(B_{0}\right)\right) \leq-k \beta \gamma-\beta \gamma_{0} \leq \beta \gamma .
$$

If $\gamma_{0} \geq 0$, let $k^{\prime}>0$ be the smallest integer such that $k^{\prime} \gamma>\gamma_{0}$, let $C_{n}^{\prime}=$ 
$B_{0} \cup A_{n} \cup \ldots \cup A_{n+k^{\prime}-1}, D_{n}^{\prime}=A_{0} \cup B_{n} \cup \ldots \cup B_{n+k^{\prime}-1}$. For large $\mathrm{n}, \lambda\left(C_{n}^{\prime}\right)>$ $\lambda\left(D_{n}^{\prime}\right)$, so $\sigma\left(C_{n}^{\prime}\right) \geq \sigma\left(D_{n}^{\prime}\right)$. It follows that

$$
\sigma\left(A_{0}\right)-\sigma\left(B_{0}\right)-\beta\left(\lambda\left(A_{0}\right)-\lambda\left(B_{0}\right)\right) \leq k^{\prime} \beta \gamma-\beta \gamma_{0} \leq \beta \gamma_{0}
$$

Together, (3.14) and (3.15) give (3.13) and $\left\|T_{\beta}\right\|_{1} \leq \beta \gamma$. If $\Gamma(H)$ $=\gamma \mathbb{Z}$, the proof is complete. If $\Gamma(H)=\mathbb{R}$, then $H$ is not of trace class, so the value of $\beta$ is independent of $\gamma$. Letting $\gamma \rightarrow 0$, it follows that $T_{\beta}=0$, so $Q=\left(I+e^{\beta H}\right)^{-1}$.

Example 3. 5. Suppose that $H$ has a complete orthonormal set of eigenvectors $\left\{f_{n}: n=0,1,2 \ldots\right\}$ with eigenvalues $\lambda_{n}=n$ 。 For example, the harmonic oscillator Hamiltonian $H=\frac{1}{2}\left(-\frac{d^{2}}{d t^{2}}+t^{2}-1\right)$ on $L^{2}(\mathbb{R})$ has eigenvectors

$$
f_{n}(t)=(n !)^{-1 / 2} 2^{n / 2} i^{-n} \pi^{-3 / 4} e^{2 / 2} \int u^{n} e^{-u^{2}+2 i t u} d u
$$

[5, Lemma 7.12]. Now $\Gamma(H)=\mathbb{Z}$. If $(H, Q)$ is a passive pair, then $f_{n}$ is an eigenvector of $Q$, and the eigenvalues $\rho_{n}$ satisfy either (a) $\rho_{n}=0$ for all $n \geq 3$, or (b) $0<\rho_{n}<1$ for all $n$ (Proposition 3.2). Indeed $(H, Q)$ is a passive pair if and only if either

$$
\begin{aligned}
& Q f_{0}=\rho_{0} f_{0}, Q f_{1}=\rho_{1} f_{1}, Q f_{2}=\rho_{2} f_{2}, Q f_{n}=0 \quad(n \geq 3), \\
& \text { where } 1 \geq \rho_{0} \geq \rho_{1} \geq \rho_{2} \geq 0, \rho_{0}+\rho_{1} \leq 1, \\
& \left(1-\rho_{0}\right)\left(1-\rho_{1}\right) \rho_{2} \leq \rho_{0} \rho_{1}\left(1-\rho_{2}\right), \rho_{0}\left(1-\rho_{1}\right) \rho_{2} \leq\left(1-\rho_{0}\right) \rho_{1}\left(1-\rho_{2}\right),
\end{aligned}
$$

or

(b) $Q=\left(I+e^{\beta(H+T)}\right)^{-1}$ for some $\beta \geq 0$ and some $T$ with $\|T\|_{1} \leq 1$.

The necessity of (b) if $0<\rho_{n}<1$ follows from Proposition 3.4 and the sufficiency is established by verifying (3.12) directly.

If $H$ is unbounded and $\Gamma(H)=(0)$, there may be passive pairs $(H, Q)$ where $Q$ and $I-Q$ are injective but $T_{\beta}$ is not of trace class for any $\beta \geq 0$.

Example 3.6. Suppose that the index set $A=\{0,1,2 \ldots\}$, and $\lambda_{n}=2^{n}$. Then $\Gamma(H)=(0)$, and (3.12) simplifies to

$$
\sigma_{0} \geq 0, \sigma_{n+1} \geq \sum_{i=0}^{n} \sigma_{i} \text { 。 }
$$

If $\sigma_{n}=3^{n}$, for example, this condition is satisfied, but $\sum\left|\sigma_{n}-\beta \lambda_{n}\right|=\infty$ for all $\beta$. Thus if $Q f_{n}=\left(1+e^{\sigma}\right)^{-1} f_{n}$, then $(H, Q)$ is passive, but $T_{\beta}$ 
is not of trace class.

\section{§4. Spectrally Passive Quasi-free States}

In this section, let $H$ be a Hamiltonian on the one-particle Hilbert space $\mathscr{H}$, with associated quasi-free one-parameter action $\tau$ on $\mathfrak{U}(\mathscr{H})$ given by (1.2). Let $\omega$ be a gauge-invariant quasi-free state of $\mathfrak{U}(\mathscr{H})$ with associated one-particle density $Q$, so that (1.3) is satisfied. Throughout this section, suppose that $\omega$ is spectrally passive with respect to $\tau$. In particular, $\omega$ is $\tau$-invariant, so $Q$ commutes with $H$ and its spectral measure $P$.

Lemma 4.1. Let $0 \leq m \leq n$ be integers, and $\left\{\lambda_{i}: 1 \leq i \leq n\right\}$ be real numbers such that $\sum_{i=1}^{m} \lambda_{i} \geq \sum_{j=m+1}^{n} \lambda_{j}$. Let $\left\{f_{i}: 1 \leq i \leq n\right\}$ be an orthonormal set, and suppose that $f_{i} \in P\left(\lambda_{i}, \infty\right) \mathscr{H} \quad(1 \leq i \leq m), f_{j} \in P\left(-\infty, \lambda_{j}\right) \mathscr{H} \quad(m<$ $j \leq n)$ and $\left(f_{i}, Q f_{j}\right)=0(1 \leq i<j \leq n)$. Then

$$
\prod_{i=1}^{m}\left(f_{i}, Q f_{i}\right) \prod_{j=m+1}^{n}\left(1-\left(f_{j}, Q f_{j}\right)\right) \leq \prod_{i=1}^{m}\left(1-\left(f_{i}, Q f_{i}\right)\right) \prod_{j=m+1}^{n}\left(f_{j}, Q f_{j}\right) .
$$

If $\sum_{i=1}^{m} \lambda_{i}>\sum_{j=m+1}^{n} \lambda_{j}, \quad$ and $f_{i} \in P\left[\lambda_{i}, \infty\right) \mathscr{H} \quad(1 \leq i \leq m), f_{j} \in P\left(-\infty, \lambda_{j}\right] \mathscr{H}$ $(m<j \leq n)$, then (4.1) remains valid.

Proof. The proof is quite similar to [4, Lemma 3.8]. Consider $x=a\left(f_{1}\right) a\left(f_{2}\right) \ldots a\left(f_{m}\right) a^{*}\left(f_{m+1}\right) a^{*}\left(f_{m+2}\right) \ldots a^{*}\left(f_{n}\right) \in R(-\infty, 0)$.

Then (1.3), the canonical anticommutation relations, the orthonormality and the condition $\left(f_{i}, Q f_{j}\right)=0$ give

$$
\begin{aligned}
& \omega\left(x^{*} x\right)=\prod_{i=1}^{m}\left(f_{i}, Q f_{i}\right) \prod_{j=m+1}^{n}\left(1-\left(f_{j}, Q f_{j}\right)\right) \\
& \omega\left(x x^{*}\right)=\prod_{i=1}^{m}\left(1-\left(f_{i}, Q f_{i}\right)\right) \prod_{j=m+1}^{n}\left(f_{j}, Q f_{j}\right) .
\end{aligned}
$$

The condition (1.1) defining spectral passivity, gives (4.1).

The final statement follows on perturbing $\lambda_{i}$ slightly.

Now write $\mathscr{H}=\mathscr{H}_{p} \oplus \tilde{\mathscr{H}}, H=H_{p} \oplus \tilde{H}, Q=Q_{p} \oplus \tilde{Q}$, where $\mathscr{H}_{p}$ is the closed linear span of the eigenspaces of $H$. Identify $\mathfrak{A}(\tilde{\mathscr{H}})$ with the $C^{*}$-subalgebra of $\mathfrak{U}(\mathscr{H})$ generated by $\{a(f): f \in \tilde{\mathscr{H}}\}$. The restriction 
of $\omega$ to $\mathfrak{X}(\tilde{\mathscr{H}})$ is the gauge-invariant quasi-free state with oneparticle density $\tilde{Q}$, and it is spectrally passive for the restriction of $\tau$ to $\mathfrak{A}(\tilde{\mathscr{H}})$. This restricted action is the quasi-free action with oneparticle Hamiltonian $\tilde{H}$, which has no eigenvectors. The results of de Cannière [4] may therefore be applied to $\tilde{H}, \tilde{Q}$. He proved (in a slightly different formulation) that either $\tilde{Q}=\tilde{P}(-\infty, \mu)$ for some $-\infty \leq \mu \leq \infty$, or $\tilde{Q}=\left(\tilde{I}+e^{\beta \tilde{H}-\tilde{\mu}}\right)^{-1}$ for some $\beta \geq 0$ and some $-\infty<\mu$ $<\infty$, where $\tilde{P}$ is the spectral measure of $\tilde{H}$, and $\tilde{I}$ is the identity operator on $\tilde{\mathscr{H}}$. He also stated without proof [4, Remark 3.14] that in fact $\tilde{Q}=\tilde{P}(-\infty, 0)$ or $\tilde{Q}=\left(\tilde{I}+e^{\beta \tilde{H}}\right)^{-1}$. This last fact is contained in the following lemma, which also removes some superfluous conditions from [4, Lemma 4.3]. The lemma could be proved in a similar fashion to [4], using the known characterization of the KMS states of quasi-free actions and the inequality

$$
\omega\left(x^{*} x\right) \leq e^{\beta \lambda} \omega\left(x x^{*}\right) \quad(x \in R(-\infty, \lambda)),
$$

valid for KMS states $\omega$ at inverse temperature $\beta$ [3]. However, it is no great hardship to give a proof from first principles.

Lemma 4. 2. Suppose that $\tilde{\mathscr{H}} \neq(0)$.

1. If $\tilde{Q}=\tilde{P}(-\infty, \mu)$ for some $-\infty \leq \mu \leq \infty$, then $\tilde{Q}=\tilde{P}(-\infty, 0)$.

2. If $\tilde{Q}=\left(\tilde{I}+e^{\beta \tilde{H}-\mu \tilde{I}}\right)^{-1}$ for some $\beta \geq 0$ and $-\infty<\mu<\infty$, then $\mu=0$ and $Q=\left(\tilde{I}+e^{\beta H}\right)^{-1}$.

Proof. 1. For unit vectors $f$ in $\tilde{P}(0, \infty) \tilde{\mathscr{H}}, g$ in $\tilde{P}(-\infty, 0) \tilde{\mathscr{H}}$, Lemma 4.1 with $n=1$ gives

$$
\begin{aligned}
(f, Q f) & \leq 1-(f, Q f) \\
1-(g, Q g) & \leq(g, Q g) .
\end{aligned}
$$

The result follows immediately.

2. Let $S$ be the set of all real numbers $\lambda$ such that $(\lambda-\varepsilon, \lambda)$ and $(\lambda, \lambda+\varepsilon)$ intersect the spectrum of $H$ for all $\varepsilon>0$. Then $S$ is uncountable and dense in itself, and generates a dense subgroup of $\mathbb{R}$.

Let $f_{0}$ be a normalised eigenvector of $H$ with eigenvalue $\lambda_{0}$. For any $\varepsilon>0$, there exist integers $0 \leq m \leq n$ and distinct $\lambda_{i}$ in $S(1 \leq i \leq n)$, not equal to $\lambda_{0}$, such that

$$
0<\lambda_{0}+\sum_{i=1}^{m} \lambda_{i}-\sum_{j=m+1}^{n} \lambda_{j}<\varepsilon_{0}
$$


For $0<\eta<\min \left\{\frac{1}{2}\left|\lambda_{i}-\lambda_{j}\right|: 0 \leq i<j \leq n\right\}$, let $f_{i}$ be a unit vector in $\tilde{P}\left(\lambda_{i}, \lambda_{i}+\eta\right) \tilde{\mathscr{H}}(1 \leq i \leq m)$ or in $\tilde{P}\left(\lambda_{i}-\eta, \quad \lambda_{i}\right) \tilde{\mathscr{H}}(m<i \leq n)$. Applying Lemma 4.1,

$$
\left(f_{0}, Q f_{0}\right) \prod_{i=1}^{m} \frac{\left(f_{i}, Q f_{i}\right)}{1-\left(f_{i}, Q f_{i}\right)} \leq\left(1-\left(f_{0}, Q f_{0}\right)\right) \prod_{j=m+1}^{n} \frac{\left(f_{j}, Q f_{j}\right)}{1-\left(f_{j}, Q f_{j}\right)}
$$

But

$$
\begin{array}{ll}
\frac{\left(f_{i}, Q f_{i}\right)}{1-\left(f_{i}, Q f_{i}\right)} \geq \exp \left(-\beta\left(\lambda_{i}+\eta\right)+\mu\right) & (1 \leq i \leq m) \\
\frac{\left(f_{j}, Q f_{j}\right)}{1-\left(f_{j}, Q f_{j}\right)} \leq \exp \left(-\beta\left(\lambda_{j}-\eta\right)+\mu\right) & (m<j \leq n)
\end{array}
$$

so

$$
\begin{aligned}
\left(f_{0}, Q f_{0}\right) \exp \{-\beta & \left.\sum_{i=1}^{m} \lambda_{i}+m(\mu-\beta \eta)\right\} \\
& \leq\left(1-\left(f_{0}, Q f_{0}\right)\right) \exp \left\{-\beta \sum_{j=m+1}^{n} \lambda_{j}+(n-m)(\beta \eta+\mu)\right\} .
\end{aligned}
$$

Letting $\eta \rightarrow 0$,

$$
\begin{aligned}
\left(f_{0}, Q f_{0}\right) & \leq\left(1-\left(f_{0}, Q f_{0}\right)\right) \exp \left\{\beta\left(\sum_{i=1}^{m} \lambda_{i}-\sum_{j=m+1}^{n} \lambda_{j}\right)+(n-2 m) \mu\right\} \\
& \leq\left(1-\left(f_{0}, Q f_{0}\right)\right) \exp \left\{\beta\left(\varepsilon-\lambda_{0}\right)+(n-2 m) \mu\right\}
\end{aligned}
$$

A similar argument, excluding $f_{0}$ and $\lambda_{0}$, shows that

$$
\lambda_{i} \in S \quad(1 \leq i \leq n), 0<\sum_{i=1}^{m} \lambda_{i}-\sum_{j=m+1}^{n} \lambda_{j}<\varepsilon \Rightarrow \exp (\beta \varepsilon+(n-2 m) \mu) \geq 1 .
$$

Since $S$ is uncountable, one may find such $\lambda_{i}$ with $2 m-n$ arbitrary (see [4, Lemma 3.16]), and it follows that $\mu=0$.

Now in (4.2), letting $\varepsilon \rightarrow 0$,

$$
\left(f_{0}, Q f_{0}\right) \leq\left(1+e^{\beta \lambda_{0}}\right)^{-1} \text {. }
$$

Similarly choosing $0 \leq k \leq l$, distinct $\mu_{i}(1 \leq i \leq l)$ in $S$, not equal to $\mu_{0}=\lambda_{0}$, such that

$$
0<-\lambda_{0}+\sum_{i=1}^{k} \mu_{i}-\sum_{j=k+1}^{l} \mu_{j}<\varepsilon
$$

and unit vectors $g_{i}$ in $\tilde{P}\left(\mu_{i}, \mu_{i}+\eta\right) \tilde{\mathscr{H}}(1 \leq i \leq k), g_{j}$ in $\tilde{P}\left(\mu_{j}-\eta, \mu_{j}\right) \tilde{\mathscr{H}}$ $(k<j \leq l)$ where $0<\eta<\min \left\{\frac{1}{2}\left|\mu_{i}-\mu_{j}\right|: 0 \leq i<j \leq l\right\}$, and applying Lemma 4.1 to $g_{1}, \ldots g_{l}, f_{0}$ gives

$$
1-\left(f_{0}, Q f_{0}\right) \leq\left(f_{0}, Q f_{0}\right) \exp \left\{\beta\left(\sum_{i=1}^{m} \mu_{i}-\sum_{j=k+1}^{l} \mu_{j}\right)\right\}
$$




$$
\leq\left(f_{0}, Q f_{0}\right) e^{\beta\left(\lambda_{0}+\varepsilon\right)}
$$

and hence $\left(f_{0}, Q f_{0}\right) \geq\left(1+e^{\beta \lambda_{0}}\right)^{-1}$. Thus $Q_{p}=\left(I_{\mathscr{H}_{p}}+e^{\beta H_{p}}\right)^{-1}$, so $Q=(I$ $\left.+e^{\beta H}\right)^{-1}$.

Remark. If $\tilde{\mathscr{H}} \neq(0)$, the Connes spectrum of $\tau$ is $\mathbb{R}$. (I am grateful to A. Kishimoto for confirmation of this.) Thus Lemma 4.2(2, is analogous to Proposition 3.4(2)。

Theorem 4.3. Lel $H$ be the one-particle Hamiltonian of a oneparameter group $\tau$ of quasi-free automorphisms of $\mathscr{A}(\mathscr{H}), P$ be the spectral measure of $H$, and $\omega$ be a gauge-invariant quasi-free state of $\mathfrak{A}(\mathscr{H})$ which is spectrally passive for $\tau$. The one-particle density $Q$ of $\omega$ satisfies one of the following conditions:

(i) $Q=\left(I+e^{\beta H}\right)^{-1}$ for some $\beta \geq 0$,

(ii) $Q(I-P(0))=P(-\infty, 0)$,

(iii) $Q$ and $I-Q$ are injective, and $(H, Q$ ) is a passive pair (in particular, $H$ and $Q$ are diagonalisable $)$,

(iv) There is a decomposition $\mathscr{H}=\mathscr{H}_{1} \oplus \mathscr{H}_{2}, H=H_{1} \oplus H_{2}, Q=Q_{1} \oplus$ $P_{2}(-\infty, 0)$ where $H_{1}$ is of trace class, $\left(-\left\|H_{1}\right\|_{1},\left\|H_{1}\right\|_{1}\right)$ is contained in the resolvent set of $H_{2}, Q_{1}$ and $I_{\mathscr{H}_{1}}-Q_{1}$ are invertible and $\left(H_{1}, Q_{1}\right)$ is a passive pair. (Here, $P_{2}$ is the spectral measure of $H_{2 \cdot}$ )

Proof. By Lemma 4.2 and the preceding remarks, either $Q=(I$ $\left.+e^{\beta H}\right)^{-1}$ in which case (i) is satisfied, or $\tilde{Q}=\tilde{P}(-\infty, 0)$. In the remainder of the proof, assume that $\tilde{Q}=\tilde{P}(-\infty, 0)$. Assume also that (ii) is not satisfied, so that there is a unit vector $f_{0}$ in $P(0, \infty) \mathscr{H}$ with $\left(f_{0}, Q f_{0}\right)>0$ or a unit vector $g_{0}$ in $P(-\infty, 0)$ with $\left(g_{0}, Q g_{0}\right)<1$. Let $c=\left(f_{0}, Q f_{0}\right)^{-1}-1$ or $c=\left(1-\left(g_{0}, Q g_{0}\right)\right)^{-1}-1$, respectively.

For real $\lambda$, let $\mathscr{H}_{\lambda}=P(\lambda) \mathscr{H}, Q_{\lambda}=Q \mid \mathscr{H}_{\lambda \circ}$. Let $\left\{f_{i}: 1 \leq i \leq 2 m\right\}$ be an orthonormal set in $\mathscr{H}_{\lambda}$ such that $\left(f_{i}, Q f_{j}\right)=0(1 \leq i<j \leq 2 m)$. Applying Lemma 4.1 to $f_{0}, f_{1}, \ldots \int_{2 m}$ or $f_{1}, \ldots f_{2 m}, g_{0}$, gives (2.1) where $n=$ $2 m$. By Lemma 2.2(2), $\mathscr{H}_{\lambda}$ has a complete orthonormal set of eigenvectors for $Q_{\lambda}$. It follows that $\mathscr{H}_{p}$ has a complete orthonormal set of common eigenvectors for $H_{p}, Q_{p}$, and Lemma 4.1 shows that $\left(H_{p}\right.$, $Q_{p}$ ) is a passive pair. Since (ii) is not satisfied, the pair is non- 
trivial. By Proposition 3.2, there is a decomposition $\mathscr{H}_{p}=\mathscr{H}_{1} \oplus \mathscr{H}_{3}$, $H_{p}=H_{1} \oplus H_{3}, Q_{p}=Q_{1} \oplus P_{3}(-\infty, 0)$, where $\left(-\left\|H_{1}\right\|_{1},\left\|H_{1}\right\|_{1}\right)$ is contained in the resolvent set of $H_{3}, Q_{1}$ and $I_{\mathscr{H}_{1}}-Q_{1}$ are injective, and $\left(H_{1}, Q_{1}\right)$ is a passive pair. $\left(\mathscr{H}_{3}\right.$ may be $(0)$, in which case $\left\|H_{1}\right\|_{1}$ may be infinite.) If $\tilde{\mathscr{H}}=(0)$, the proof is now complete, since either (iii) applies (if $\mathscr{H}_{3}=(0)$ ) or (iv) applies with $\mathscr{H}_{2}=\mathscr{H}_{3 \circ}$

If $\tilde{\mathscr{H}} \neq(0)$ let $\gamma=\inf \{|\lambda|: \lambda \tilde{I}-\tilde{H}$ is not invertible $\}$. It suffices to show that $\left\|H_{1}\right\|_{1} \leq \gamma$, for then $\mathscr{H}_{2}$ may be taken to be $\mathscr{H}_{3} \oplus \tilde{\mathscr{H}}$. Suppose, on the contrary, that there is an orthonormal set $\left\{f_{i}: 1 \leq i \leq\right.$ $n\}$ in $\mathscr{H}_{1}$, an integer $0 \leq m \leq n$, and scalars $\lambda_{i}, \rho_{i}$ such that $0<\rho_{i}<1$, $H_{i} f_{i}=\lambda_{i} f_{i}, Q f_{i}=\rho_{i} f_{i} \quad(1 \leq i \leq n), \sum_{i=1}^{m} \lambda_{i}-\sum_{j=m+1}^{n} \lambda_{j}=\gamma^{\prime}>\gamma$. There is a unit vector $\tilde{f}_{0}$ in $\tilde{P}\left(-\gamma^{\prime},-\gamma\right) \tilde{\mathscr{H}}$ or a unit vector $\tilde{g}_{0}$ in $\tilde{P}\left(\gamma, \gamma^{\prime}\right) \tilde{\mathscr{H}}$. By assumption $\left(\tilde{f}_{0}, Q \tilde{f}_{0}\right)=1$ or $\left(\tilde{g}_{0}, Q \tilde{g}_{0}\right)=0$. Applying Lemma 4.1 to $\tilde{f}_{0}, f_{1}, f_{2}, \ldots f_{n}$ or to $f_{1}, f_{2}, \ldots f_{n}, \tilde{g}_{0}$, gives

$$
\prod_{i=1}^{m} \rho_{i} \prod_{j=m+1}^{n}\left(1-\rho_{j}\right) \leq 0 \text {. }
$$

This contradicts the fact that $0<\rho_{i}<1$.

\section{§5. Quasi-free States of Passive Pairs}

This section is devoted to establishing that if $Q$ satisfies any of the conditions (i) - (iv) of Theorem 4.3, then the associated quasifree state $\omega$ is spectrally passive.

If condition (i) is satisfied, then $\omega$ is the unique KMS state at inverse temperature $\beta[2,5.2 .23]$, and is therefore spectrally passive [3]. If condition (ii) is satisfied, then $\omega$ is a ground state $[2,5.3$. $20]$, so that $\omega\left(x^{*} x\right)=0 \leq \omega\left(x x^{*}\right)$ for $x$ in $R(-\infty, 0)$. Thus $\omega$ is spectrally passive.

The remaining conditions (iii) and (iv) are both covered by the following result, where $\mathscr{H}_{2}$ may be $(0)$, in which case the resolvent set of $H_{2}$ is $\boldsymbol{R}$, and $\left\|H_{1}\right\|_{1}$ may be infinite (condition (iii) of Theorem 4.3).

Theorem 5.1. Suppose that $\mathscr{H}=\mathscr{H}_{1} \oplus \mathscr{H}_{2}, \quad H=H_{1} \oplus H_{2}, \quad Q=Q_{1} \oplus$ $P_{2}(-\infty, 0)$, where $P_{2}$ is the spectral measure of $H_{2},\left(H_{1}, Q_{1}\right)$ is a passive pair and $\left(-\left\|H_{1}\right\|_{1},\left\|H_{1}\right\|_{1}\right)$ is contained in the resolvent set of $H_{2}$. Then the gauge-invariant quasi-free state $\omega$ with one-particle density $Q$ is spec- 
trally passive with respect to the one-parameter group of quasi-free automorphisms of $\mathfrak{U}(\mathscr{H})$ with one-particle Hamiltonian $H$.

Proof. First, suppose that $\mathscr{H}_{1}$ is finite-dimensional with an orthonormal basis $\left\{f_{i}: 1 \leq i \leq n\right\}$ of common eigenvectors for $H_{1}, Q_{1}$ with eigenvalues $\lambda_{i}, \rho_{i}$ respectively. Let $\mathscr{G}$ be an orthonormal basis of $\mathscr{H}_{2}$. There is a standard construction $[2,5.2 .5]$, by which $\mathfrak{A}\left(\mathscr{H}_{2}\right)$ is identified with $\underset{\mathscr{G}}{\otimes} M_{2}$ and $\mathfrak{U}(\mathscr{H})$ with $\underset{\{1, \ldots n\} \cup \mathscr{G}}{\otimes} M_{2}$ and hence with the $C^{*}$-algebra of $2^{n} \times 2^{n}$ matrices with entries in $\mathfrak{A}\left(\mathscr{H}_{2}\right)$. Any integer $1 \leq j \leq 2^{n}$ has a binary representation $j=1+\sum_{r \in S_{j}} 2^{r-1}$ for some subset $S_{j}$ of $\{1, \ldots n\}$. The identification is made in such a way that

$$
\tau_{t}(x)=\left[\exp \left(i t\left(\lambda\left(S_{j}\right)-\lambda\left(S_{k}\right)\right)\right) \tau_{t}^{(2)}\left(x_{j k}\right)\right]_{1 \leq j, k \leq 2^{n}}
$$

for any $x=\left[x_{j k}\right]$ in $\mathscr{U}(\mathscr{H})$, where $\tau^{(2)}$ is the one-parameter quasi-free action on $\mathfrak{U}\left(\mathscr{H}_{2}\right)$ with one-particle Hamiltonian $H_{2}$ If $\mathfrak{A}\left(\mathscr{H}_{2}\right)$ is identified with the $C^{*}$-subalgebra of $\mathfrak{U}(\mathscr{H})$ generated by $\{a(f)$ : $\left.f \in \mathscr{H}_{2}\right\}$, then $\tau^{(2)}$ is the restriction of $\tau$. Furthermore

$$
\omega(x)=\sum_{j=1}^{2^{n}} \theta_{j} \omega_{2}\left(x_{j j}\right)
$$

where

$$
\begin{aligned}
& \theta_{j}=\prod_{i \in S_{j}} \rho_{i} \prod_{i \in S_{j}^{\prime}}\left(1-\rho_{i}\right) \\
& S_{j}^{\prime}=\{1, \ldots n\} \backslash S_{j}
\end{aligned}
$$

and $\omega_{2}$ is the ground state for $\tau_{2}$ (the gauge-invariant quasi-free state with one-particle density $\left.P_{2}(-\infty, 0)\right)$. These facts may be verified by induction on the dimension $n$ of $\mathscr{H}_{1}$, using the results of [4, p. 142] for $n=1$.

It follows from (5.1) that

$$
x \in R(-\infty, 0) \Leftrightarrow x_{j k} \in R\left(-\infty, \lambda\left(S_{k}\right)-\lambda\left(S_{j}\right)\right) \text { for all } j, k \text { 。 }
$$

(The spectral spaces for $\tau^{(2)}$ are the parts of the spectral spaces for $\tau$ which are contained in $\left.\mathfrak{U}\left(\mathscr{H}_{2}\right)_{\text {. }}\right)$ It is therefore sufficient to show that the condition (5.2) implies that

$$
\sum_{j, k} \theta_{k} \omega_{2}\left(x_{j k}^{*} x_{j k}\right) \leq \sum_{j, k} \theta_{j} \omega_{2}\left(x_{j k} x_{j k}^{*}\right) \text {. }
$$

Considering one coordinate $x_{j k}$ at a time, it suffices to show that 
$\theta_{k} \omega_{2}\left(x^{*} x\right) \leq \theta_{j} \omega_{2}\left(x x^{*}\right)$ for all $x$ in $R\left(-\infty, \lambda\left(S_{k}\right)-\lambda\left(S_{j}\right)\right) \cap \mathfrak{A}\left(\mathscr{H}_{2}\right)$.

If $\lambda\left(S_{k}\right) \leq \lambda\left(S_{j}\right),(5.4)$ follows immediately from the fact that $\omega_{2}$ is a ground state. If $\lambda\left(S_{k}\right)>\lambda\left(S_{j}\right)$ then $\theta_{k} \leq \theta_{j}$ by (3.1) applied to $S_{k} \backslash S_{j}, S_{j} \backslash S_{k}$. Since $\lambda\left(S_{k}\right)-\lambda\left(S_{j}\right) \leq\left\|H_{1}\right\|_{1}$, it suffices to show that

$$
\omega_{2}\left(x^{*} x\right) \leq \omega_{2}\left(x x^{*}\right) \text { for all } x \text { in } R\left(-\infty,\left\|H_{1}\right\|_{1}\right) \cap \mathfrak{X}\left(\mathscr{H}_{2}\right) .
$$

Since $\left(-\left\|H_{1}\right\|_{1},\left\|H_{1}\right\|_{1}\right)$ is contained in the resolvent set of $H_{2}$ and $\omega_{2}$ is a ground state, it can be shown as in [4, p. 146] that

$$
\omega_{2}\left(x^{*} x\right)=\left|\omega_{2}(x)\right|^{2} \leq \omega_{2}\left(x x^{*}\right)
$$

as required.

Next, suppose that $\mathscr{H}_{1}$ is infinite-dimensional with a complete orthonormal set $\mathscr{F}$ of common eigenvectors for $H_{1}, Q_{1}$. For each finite subset $F$ of $\mathscr{F}$, let $\mathscr{H}_{F}$ be the linear span of $F \cup \mathscr{H}_{2}$. Then $\mathfrak{A}\left(\mathscr{H}_{F}\right)$ is a $\tau$-invariant $C^{*}$-subalgebra of $\mathfrak{U}(\mathscr{H})$, the restriction of $\tau$ to $\mathfrak{A}\left(\mathscr{H}_{F}\right)$ is the qausi-free action with one-particle Hamiltonian $H \mid \mathscr{H}_{F}$, and the restriction of $\omega$ is the gauge-invariant quasi-free state with oneparticle density $Q \mid \mathscr{H}_{F}$. By the finite-dimensional case above, $\omega\left(x^{*} x\right)$ $\leq \omega\left(x x^{*}\right)$ for all $x$ in $R(-\infty, 0) \cap \mathfrak{A}\left(\mathscr{H}_{F}\right)$. Since $R(-\infty, 0)=\left[\cup_{F}\right.$ $\left.\left(R(-\infty, 0) \cap \mathfrak{A}\left(\mathscr{H}_{F}\right)\right)\right]^{-}$, it follows that $\omega$ is spectrally passive.

Corollary 5.2. Let $H$ be the one-particle Hamiltonian of a oneparameter quasi-free action $\tau$ on $\mathfrak{X}(\mathscr{H})$, and $Q$ be the one-particle density of a gauge-invariant quasi-free state $\omega$ on $\mathfrak{U}(\mathscr{H})$, and suppose that any of the conditions (i), (ii), (iii) and (iv) of Theorem 4.3 is satisfied. Then $\omega$ is spectrally passive for $\tau$.

Proof. Cases (i) and (ii) were discussed at the beginning of this section. Case (iii) follows from Theorem 5.1 with $\mathscr{H}_{2}=(0)$. Case (iv) is also covered by Theorem 5.1 .

\section{§ 6. The Gauge-invariant CAR Algebra}

The $\mathrm{C}^{*}$-algebra of primary concern in [4] was $\mathfrak{Y}(\mathscr{H})^{(0)}$, and the corresponding results for $\mathscr{U}(\mathscr{H})$ were merely stated. Here, detailed arguments have been given for $\mathfrak{A}(\mathscr{H})$, but it is possible to modify 
them for the gauge-invariant part. The main technical difference is that if $\omega$ is merely assumed to be spectrally passive on $\mathscr{A}(\mathscr{H})^{(0)}$, then (4.1) can only be deduced if $n=2 \mathrm{~m}$. A correspondingly weaker notion of passive pairs is needed.

The pair $(H, Q)$ is weakly passive if $\mathscr{H}$ has a complete orthonormal set of common eigenvectors with eigenvalues $\lambda_{\alpha}, \rho_{\alpha}$, and (3.1) holds for any finite subsets $A_{1}, A_{2}$ of $A$ such that $\lambda\left(A_{1}\right)>\lambda\left(A_{2}\right)$ and $\left|A_{1}\right|=\left|A_{2}\right|$, where $|B|$ denotes the cardinality of $B$.

The methods of Sections 4 and 5 now give the following modification of Theorem 4.3 and Corollary 5.2.

Theorem 6.1. Let $H$ be the one-particle Hamiltonian of a one-parameter quasi-free action $\tau, P$ be the spectral measure of $H$, and $Q$ be the one-particle density of a gauge-invariant quasi-free state $\omega$. The restriction of $\omega$ to $\mathfrak{A}(\mathscr{H})^{(0)}$ is spectrally passive with respect to the restriction of $\tau$ if and only if at least one of following conditions is satisfied:

( $i$ ) $Q=\left(I+e^{\beta H-\mu I}\right)^{-1}$ for some $\beta \geq 0,-\infty<\mu<\infty$,

(ii) $Q(I-P(\mu))=P(-\infty, \mu)$ for some $-\infty<\mu<\infty$,

(iii) $Q$ and $I-Q$ are injective, and $(H, Q)$ is a weakly passive pair,

(iv) There exist $-\infty \leq \mu_{1}<\mu_{2} \leq \infty$ and a decomposition $\mathscr{H}=\mathscr{H}_{1} \oplus$ $\mathscr{H}_{2}, H=H_{1} \oplus H_{2}, Q=Q_{1} \oplus P_{2}\left(-\infty, \mu_{2}\right)$ such that $Q_{1}$ and $I_{\mathscr{H}_{1}}-$ $Q_{1}$ are injective, $\left(H_{1}, Q_{1}\right)$ is a weakly passive pair, $\left(\mu_{1}, \mu_{2}\right)$ is contained in the resolvent set of $\mathrm{H}_{2}$, and the complete family $\left\{\lambda_{\alpha}\right.$ : $\alpha \in A\}$ of eigenvalues of $H_{1}$ satisfies

$$
\mu_{1} \leq \lambda\left(A_{1}\right)-\lambda\left(A_{2}\right) \leq \mu_{2}
$$

for any finite subsets $A_{1}, A_{2}$ of $A$ with $\left|A_{1}\right|=\left|A_{2}\right|+1$.

(v) $Q=c I$ for some $0 \leq c \leq 1$.

Remark. In case (iv), it can be assumed that $\mu_{1}$ or $\mu_{2}$ is finite (otherwise, case (iii) applies). Then, if $\mathscr{H}_{1}$ does not have finite even dimension, $(6.1)$ is equivalent to the condition that

$$
\left\|H_{1}-\mu I_{H_{1}}\right\|_{1} \leq \min \left(\mu_{2}-\mu, \mu-\mu_{1}\right)
$$

for some $\mu_{2} \leq \mu \leq \mu_{1}$. If $\mathscr{H}_{1}$ has dimension $2 m$, and the eigenvalues of $H_{1}$ are $\lambda_{1} \leq \lambda_{2} \leq \ldots \leq \lambda_{2 m}$, then $(6,1)$ is equivalent to the conditions that

$$
\left\|H_{1}-\lambda_{m} I_{\mathscr{H}_{1}}\right\|_{1} \leq \mu_{2}-\lambda_{m} \text { and }\left\|H_{1}-\lambda_{m+1} I_{\mathscr{H}_{1}}\right\|_{1} \leq \lambda_{m+1}-\mu_{1}
$$


Finally, with the help of Lemma 2.2(2), the results of Section 3 can be modified to describe weakly passive pairs. Let $\Gamma(H)^{(0)}$ be the closed subgroup of $\mathbb{R}$ containing those $r$ such that there are sequences of mutually disjoint finite subsets $A_{n}, B_{n}$ of $A$ such that $\left|A_{n}\right|=\left|B_{n}\right|$ and $\lambda\left(A_{n}\right)-\lambda\left(B_{n}\right) \rightarrow \gamma$.

Proposition 6.2. Let $(H, Q)$ be a weakly passive pair, and suppose that $Q$ and $I-Q$ are injective.

1. If $H-\lambda I$ is of trace class for some real $\lambda$, but $H \neq \lambda I$, then $\log \left(b\left(Q^{-1}-I\right)\right)$ is of trace class for some $b>0$, and for any $\beta>0$, $Q=\left(I+e^{\beta(H+T)-\mu I}\right)^{-1}$ for some real $\mu$ and some operator $T$ of trace class.

2. If $\Gamma(H)^{(0)}=\gamma Z$ for some $\gamma>0$, then $Q=\left(I+e^{\beta(H+T)-\mu I}\right)^{-1}$ for some $\beta \geq 0$, some real $\mu$, and some operator $T$ with $\|T\|_{1} \leq \gamma$.

3. If $\Gamma(H)^{(0)}=\mathbb{R}$, then $Q=\left(I+e^{\beta H-\mu I}\right)^{-1}$ for some $\beta \geq 0$ and some real $\mu$.

Remark. If $H$ is diagonalisable, $\Gamma(H)^{(0)}$ is the Connes spectrum of $\tau \mid \mathfrak{A}(\mathscr{H})^{(0)}$. If $H$ is not diagonalisable, the Connes spectrum is R. (I am grateful to A. Kishimoto for confirmation of this.)

\section{References}

[1] Batty, G. J. K., The KMS condition and passive states, J. Functional Analysis, 46 (1982), 246-257.

[2] Bratteli, O., and Robinson, D.W., Operator algebras and quantum statistical mechanics II, Springer-Verlag, Berlin-Heidelberg-New York, 1981.

[3] De Cannière, J., A spectral characterization of KMS states, Commun. Math. Phys. 84 (1982), 187-205.

[4] ., Passive quasi-free states of the non-interacting Fermi gas, J. Functional Analysis 58 (1984), 125-149.

[5] Davies, E. B., One-parameter semigroups, Academic Press, London-New York, 1980.

[6] Kishimoto, A., and Robinson, D. W., Derivations, dynamical systems, and spectral resolutions, Math. Scand. 56 (1985), 83-95.

[7] Pusz, W., and Woronowicz, S. L., Passive states and KMS states for general quantum systems, Commun. Math. Phys. 58 (1978), 273-290. 